\title{
Modular Neural Network and Classical Reinforcement Learning for Autonomous Robot Navigation: Inhibiting Undesirable Behaviors
}

\author{
Eric A. Antonelo, Albert-Jan Baerveldt, Thorsteinn Rögnvaldsson, and Mauricio Figueiredo
}

\begin{abstract}
Classical reinforcement learning mechanisms and a modular neural network are unified to conceive an intelligent autonomous system for mobile robot navigation. The conception aims at inhibiting two common navigation deficiencies: generation of unsuitable cyclic trajectories and ineffectiveness in risky configurations. Different design apparatuses are considered to compose a system to tackle with these navigation difficulties, for instance: 1) neuron parameter to simultaneously memorize neuron activities and function as a learning factor, 2) reinforcement learning mechanisms to adjust neuron parameters (not only synapse weights), and 3) a inner-triggered reinforcement. Simulation results show that the proposed system circumvents difficulties caused by specific environment configurations, improving the relation between collisions and captures.
\end{abstract}

\section{INTRODUCTION}

$\mathrm{R}$ obot navigation has caused frequent admiration in human beings, certainly because of the intriguing capabilities announced (and sometimes confirmed). Besides, it is easily associated to a prosperous and intense technical progress. A vast range of applications are foreseen, e.g., surveillance, rescue, and space exploration.

Despite the great appeal, it is not easy to conceive a navigation system, being considered one of the main challenges in artificial system research. Different approaches are adopted for designing navigation systems, each of which more suitable to some classes of environments and tasks.

Traditional control techniques are not suitable for designing such systems because of the huge difficulty for modeling physically the problem. The unstructured character of the environment (including the simplest one) and the nonholonomic character of common robots are among the critical points to overcome.

On the other hand, navigation systems based on computational intelligence techniques (neural networks [1], fuzzy systems, evolutionary computation [2] and swarm intelligence [3]) have reached important results. These systems show desirable characteristics, e.g., robustness and;

This work is supported by the Programme Alßan, the European Union Programme of High Level Scholarships for Latin America, scholarship no. E04M027421BR.

E. Antonelo, A.-J. Baerveldt and T. Rögnvaldsson are with the School of Information Science, Computer and Electrical Engineering, Halmstad University, Sweden (eric.antonelo@gmail.com, albert@ide.hh.se and denni@ide.hh.se, respectively).

M. Figueiredo is with Department of Computer Science, State University of Maringá, Brazil (mauricio@din.uem.br). learning and adaptation capacities.

Systems can be divided in two main classes: deliberative and reactive. Deliberative systems take decisions considering all information captured (or stored), including past information. In general such systems consider internal maps of the environment and the trajectory is planned completely a priori. Reactive systems take decisions based only on sensory information captured currently.

A special feature, the learning capability, allows identifying a distinct and important class, namely, autonomous navigation systems (considered here as those systems that learn their navigation strategies independently from the designer). These systems are of special concern to cope with inherent difficulties associated to unknown environments, e.g., unpredictability and hazardousness. The design of systems for navigating in unknown environments is even more difficult, being associated with the most interesting applications.

Most proposed intelligent autonomous systems are reactive systems [4]. Different types of navigation problems are considered. Usually autonomous systems learn two behaviors: obstacle avoidance and target seeking. Sometimes there is a specific sensory field for detecting targets [5] [6] [8]. If this is not the case, the design is more complex. In general, it is observed that systems generate undesirable behaviors, e.g., cyclic trajectories, if attractive objects are eclipsed [7].

This is the case considered in this paper: a reactive autonomous navigation system for mobile robots without a target sensory field. The main purpose of this work is to describe learning mechanisms that acquire navigation strategies that avoid undesirable behaviors. The system is composed of hierarchical modular neural networks that learn according to the classical reinforcement learning procedure [9]. Learning proceeds continuously (as the robot interacts with different classes of objects) and provides the base for acquisition of target seeking and obstacle avoidance behaviors. Despite the lack of a target sensory field, simulation results show that the autonomous system learns to generate behaviors free of cyclic trajectories and the robot efficiently avoids risky configurations.

The paper is organized as follows. Existent deficiencies of certain autonomous systems are presented in Section II. Environment and robot models are described in Section III. The following section gives an overview about the autonomous system. In Section $\mathrm{V}$ the proposed enhancements for the system are described. Section VI 
The remainder of this paper is not included as this paper is copyrighted material. If you wish to obtain an electronic version of this paper, please send an email to bib@elis.UGent.be with a request for publication P106.275.pdf. 